\title{
Measurement of the atmospheric neutrino-induced upgoing muon flux using MACRO
}

The MACRO Collaboration

M. Ambrosio ${ }^{12}$, R. Antolini ${ }^{7}$, C. Aramo ${ }^{7, n}$, G. Auriemma ${ }^{14, a}$, A. Baldini ${ }^{13}$, G. C. Barbarino ${ }^{12}$, B. C. Barish ${ }^{4}$, G. Battistoni ${ }^{6, b}$, R. Bellotti ${ }^{1}$, C. Bemporad ${ }^{13}$, P. Bernardini ${ }^{10}$, H. Bilokon ${ }^{6}$, V. Bisi ${ }^{16}$, C. Bloise ${ }^{6}$, C. Bower ${ }^{8}$, S. Bussino ${ }^{14}$, F. Cafagna ${ }^{1}$, M. Calicchio ${ }^{1}$, D. Campana ${ }^{12}$, M. Carboni ${ }^{6}$, M. Castellano ${ }^{1}$, S. Cecchini ${ }^{2, c}$, F. Cei ${ }^{11,13}$, V. Chiarella ${ }^{6}$, B. C. Choudhary ${ }^{4}$, S. Coutu ${ }^{11, o}$, L. De Benedictis ${ }^{1}$, G. De Cataldo ${ }^{1}$, H. Dekhissi ${ }^{2,17}$, C. De Marzo ${ }^{1}$, I. De Mitri ${ }^{9}$, J. Derkaoui ${ }^{2,17}$, M. De Vincenzi ${ }^{14, e}$, A. Di Credico ${ }^{7}$, O. Erriquez ${ }^{1}$, C. Favuzzi ${ }^{1}$, C. Forti ${ }^{6}$, P. Fusco ${ }^{1}$, G. Giacomelli ${ }^{2}$, G. Giannini ${ }^{13, f}$, N. Giglietto ${ }^{1}$, M. Giorgini ${ }^{2}$, M. Grassi ${ }^{13}$, L. Gray ${ }^{4,7}$, A. Grillo ${ }^{7}$, F. Guarino ${ }^{12}$, P. Guarnaccia ${ }^{1}$, C. Gustavino ${ }^{7}$, A. Habig ${ }^{3}$, K. Hanson ${ }^{11}$, A. Hawthorne ${ }^{8}$, R. Heinz ${ }^{8}$, Y. Huang ${ }^{4}$, E. Iarocci ${ }^{6, g}$, E. Katsavounidis ${ }^{4}$, I. Katsavounidis ${ }^{4}$, E. Kearns ${ }^{3}$, H. Kim ${ }^{4}$, S. Kyriazopoulou ${ }^{4}$, E. Lamanna ${ }^{14}$, C. Lane ${ }^{5}$, D. S. Levin ${ }^{11}$, P. Lipari ${ }^{14}$, N. P. Longley ${ }^{4, l}$, M. J. Longo ${ }^{11}$, F. Maaroufi ${ }^{2,17}$, G. Mancarella ${ }^{10}$, G. Mandrioli ${ }^{2}$, S. Manzoor ${ }^{2, m}$, A. Margiotta Neri ${ }^{2}$, A. Marini ${ }^{6}$, D. Martello ${ }^{10}$, A. Marzari-Chiesa ${ }^{16}$, M. N. Mazziotta ${ }^{1}$,

C. Mazzotta ${ }^{10}$, D. G. Michael ${ }^{4}$, S. Mikheyev ${ }^{4,7, h}$, L. Miller ${ }^{8}$, P. Monacelli ${ }^{9}$, T. Montaruli ${ }^{1}$, M. Monteno ${ }^{16}$, S. Mufson ${ }^{8}$, J. Musser ${ }^{8}$, D. Nicoló ${ }^{13, d}$, R. Nolty ${ }^{4}$, C. Okada ${ }^{3}$, C. Orth ${ }^{3}$, G. Osteria ${ }^{12}$, M. Ouchrif ${ }^{2,17}$,

O. Palamara ${ }^{10}$, V. Patera ${ }^{6, g}$, L. Patrizii ${ }^{2}$, R. Pazzi ${ }^{13}$, C. W. Peck ${ }^{4}$, S. Petrera ${ }^{9}$, P. Pistilli ${ }^{14, e}$, V. Popa ${ }^{2, i}$, V. Pugliese ${ }^{14}$, A. Rainó ${ }^{1}$, J. Reynoldson ${ }^{7}$, F. Ronga ${ }^{6}$, U. Rubizzo ${ }^{12}$, A. Sanzgiri ${ }^{15}$, C. Satriano ${ }^{14, a}$, L. Satta ${ }^{6, g}$, E. Scapparone ${ }^{7}$, K. Scholberg ${ }^{3}$, A. Sciubba ${ }^{6, g}$, P. Serra-Lugaresi ${ }^{2}$, M. Severi ${ }^{14}$, M. Sioli ${ }^{2}$, M. Sitta ${ }^{16}$,

P. Spinelli ${ }^{1}$, M. Spinetti ${ }^{6}$, M. Spurio ${ }^{2}$, R. Steinberg ${ }^{5}$, J. L. Stone ${ }^{3}$, L. R. Sulak ${ }^{3}$, A. Surdo ${ }^{10}$, G. Tarlé ${ }^{11}$, V. Togo

${ }^{2}$, D. Ugolotti ${ }^{2}$, M. Vakili ${ }^{15}$, C. W. Walter ${ }^{3}$, and R. Webb ${ }^{15}$.

1. Dipartimento di Fisica dell'Università di Bari and INFN, 70126 Bari, Italy

2. Dipartimento di Fisica dell'Università di Bologna and INFN, 40126 Bologna, Italy

3. Physics Department, Boston University, Boston, MA 02215, USA

4. California Institute of Technology, Pasadena, CA 91125, USA

5. Department of Physics, Drexel University, Philadelphia, PA 19104, USA

6. Laboratori Nazionali di Frascati dell'INFN, 00044 Frascati (Roma), Italy

7. Laboratori Nazionali del Gran Sasso dell'INFN, 67010 Assergi (L'Aquila), Italy

8. Depts. of Physics and of Astronomy, Indiana University, Bloomington, IN 47405, USA

9. Dipartimento di Fisica dell'Università dell'Aquila and INFN, 67100 L'Aquila, Italy

10. Dipartimento di Fisica dell'Università di Lecce and INFN, 73100 Lecce, Italy

11. Department of Physics, University of Michigan, Ann Arbor, MI 48109, USA

12. Dipartimento di Fisica dell'Università di Napoli and INFN, 80125 Napoli, Italy

13. Dipartimento di Fisica dell'Università di Pisa and INFN, 56010 Pisa, Italy

14. Dipartimento di Fisica dell'Università di Roma "La Sapienza" and INFN, 00185 Roma, Italy

15. Physics Department, Texas A\&M University, College Station, TX 77843, USA

16. Dipartimento di Fisica Sperimentale dell'Università di Torino and INFN, 10125 Torino, Italy

17. Faculty of Sciences, University Mohamed I, B.P. 424 Oujda, Morocco

a Also Università della Basilicata, 85100 Potenza, Italy $b$ Also INFN Milano, 20133 Milano, Italy

c Also Istituto TESRE/CNR, 40129 Bologna, Italy

$d$ Also Scuola Normale Superiore di Pisa, 56010 Pisa, Italy

$e$ Also Dipartimento di Fisica, Università di Roma Tre, Roma, Italy

$f$ Also Università di Trieste and INFN, 34100 Trieste, Italy

$g$ Also Dipartimento di Energetica, Università di Roma, 00185 Roma, Italy

$h$ Also Institute for Nuclear Research, Russian Academy of Science, 117312 Moscow, Russia

$i$ Also Institute for Space Sciences, 76900 Bucharest, Romania

$l$ Swarthmore College, Swarthmore, PA 19081, USA

$m$ RPD, PINSTECH, P.O. Nilore, Islamabad, Pakistan

$n$ Also INFN Catania, 95129 Catania, Italy

o Also Department of Physics, Pennsylvania State University, University Park, PA 16801, USA

(October 5, 2018)

We present a measurement of the flux of neutrino-induced upgoing muons $\left(<\mathrm{E}_{\nu}>\sim 100 \mathrm{GeV}\right)$ using the MACRO detector. The ratio of the number of observed to expected events integrated over all zenith angles is $0.74 \pm 0.036$ (stat) \pm 0.046 (systematic) \pm 0.13 (theoretical). The observed zenith distribution for $-1.0 \leq \cos \theta \leq-0.1$ does not fit well with the no oscillation expectation, giving a maximum probability for $\chi^{2}$ of $0.1 \%$. The acceptance of the detector has been extensively studied using downgoing muons, independent analyses and Monte-Carlo simulations. The other systematic uncertainties cannot be the source of the discrepancies between the data and expectations.

We have investigated whether the observed number of events and the shape of the zenith dis- 
tribution can be explained by a neutrino oscillation hypothesis. Fitting either the flux or zenith distribution independently yields mixing parameters of $\sin ^{2} 2 \theta=1.0$ and $\Delta m^{2}$ of a few times $10^{-3}$ $\mathrm{eV}^{2}$. However, the observed zenith distribution does not fit well with any expectations, giving a maximum probability for $\chi^{2}$ of $5 \%$ for the best oscillation hypothesis, and the combined probability for the shape and number of events is $17 \%$. We conclude that these data favor a neutrino oscillation hypothesis, but with unexplained structure in the zenith distribution not easily explained by either the statistics or systematics of the experiment.

\section{Submitted to Physics Letters}


Over the last decade evidence has been growing for the possibility of oscillation of atmospheric neutrinos. A first anomaly was observed in the ratio of contained muon neutrino to electron neutrino interactions in the IMB [1] and Kamiokande [2] detectors. In addition, the observation of an anomaly in the multi-GeV atmospheric neutrino ratio in Kamiokande suggested specific oscillation parameters with large mixing probability and $\Delta m^{2} \approx 10^{-2} \mathrm{eV}^{2}$ 3]. Recent results from SuperKamiokande have confirmed the anomaly in the contained event ratio and also show a strong effect in the zenith angle distribution [4] suggesting best fit parameters of $\sin ^{2} 2 \theta=1.0$ and $\Delta m^{2}$ in the range of a few times $10^{-3} \mathrm{eV}^{2}$. Also recently, the Soudan 2 detector has confirmed an anomaly in the $\mu / \mathrm{e}$ ratio of contained events using an iron-based detector [5]. Earlier results from the Frejus [6] and NUSEX [7] detectors are consistent with the expected number of contained events though with smaller statistics.

The flux of atmospheric muon neutrinos in the energy region from a few $\mathrm{GeV}$ up to hundreds of $\mathrm{GeV}$ can be inferred from measurements of upgoing muons in underground detectors. If the anomalies in the atmospheric neutrinos at lower energy are the result of neutrino oscillations, then the flux of upgoing muons should be affected both in the absolute number of events observed and in the shape of the zenith angle distribution, with relatively fewer events observed near the zenith than near the horizontal due to the longer pathlength of neutrinos near the zenith. Previous measurements of the upgoing muon flux have been made by the Baksan [8], Kamiokande [9], IMB [10] and Frejus [6] detectors with no claimed discrepancy with expectations from calculation.

The MACRO detector 11 provides an excellent tool for the study of upgoing muons. Its large area $(76.6 \mathrm{~m} \times 12 \mathrm{~m} \times 9.3 \mathrm{~m})$, fine tracking granularity (angular resolution on tracks is between $0.1^{\circ}$ and $1.0^{\circ}$ ), good time resolution (about $500 \mathrm{ps}$ ), symmetric electronics with respect to upgoing versus downgoing muons and fully-automated analysis permit detailed studies of the detector acceptance and possible sources of backgrounds to upgoing muons. In addition, the overburden of the Gran Sasso Laboratory (minimum rock overburden of $3150 \mathrm{hg} / \mathrm{cm}^{2}$ ) is significantly larger than for the locations of the previous experiments with the highest statistics on upgoing muons (Baksan and IMB). This provides additional shielding against possible sources of background induced by down-going muons.

In our first measurement on upward-going (upgoing) muons, we reported on a deficit in the total number of observed upgoing muons with respect to expectations and also an anomalous zenith angle distribution [12]. Here, we report on a data set with much higher statistics 13 which retains the same basic features as reported previously. In addition, an extensive and exhaustive study has been performed on systematic effects in the analysis and detector acceptance.

The upgoing muon data presented here come from three running periods and detector configurations: the lower half of one supermodule from March 1989 - November 1991 (1.38 effective live-years), the lower half of 6 supermodules from December 1992 - June 1993 (0.413 effective live-years) and the full detector from April 1994 - December 1997 (2.89 effective live-years). Results from the first two periods have already been published [12].

The sign of the direction that muons travel through MACRO is determined from the time-of-flight between at least two different layers of scintillator counters combined with the path length of a track reconstructed in the streamer tubes. The measured muon velocity is calculated with the convention that muons going down through the detector will be expected to have $1 / \beta$ near +1 while muons going up through the detector will be expected to have $1 / \beta$ near -1 . Several cuts are imposed to remove backgrounds caused by radioactivity in near coincidence with muons and showering events which may result in bad time reconstruction. The most important cut requires that the position of a muon hit in each scintillator as determined from the timing within the scintillator counter agrees to within $\pm 70 \mathrm{~cm}$ of the position indicated by the streamer tube track.

It has been observed that downgoing muons which pass near or through MACRO may produce low-energy, upgoing particles. These could appear to be neutrino-induced upgoing muons if the down-going muon misses the detector 14. This background has been suppressed by imposing a cut requiring that each upgoing muon must traverse at least $200 \mathrm{~g} / \mathrm{cm}^{2}$ of material in the bottom half of the detector. Finally, a large number of nearly horizontal $(\cos \theta>-0.1)$, but upgoing muons have been observed coming from azimuth angles (in local coordinates) from $30^{\circ}-50^{\circ}$. This direction corresponds to a cliff in the mountain where the overburden is insufficient to remove nearly horizontal, downgoing muons which have scattered in the mountain and appear as upgoing. We exclude this region from both our observation and MonteCarlo calculation of the upgoing events.

Figure 11 shows the $1 / \beta$ distribution for the MACRO data from the full detector running (for the older data see the equivalent figure in Ref. [12]). A clear peak of upgoing muons is evident centered on $1 / \beta=-1$. There are 398 events in the range $-1.25<1 / \beta<-0.75$ which we define as our upgoing muon sample from this data set. We combine these data with the previously published data (with 4 additional events due to an updated analysis) for a total of 479 upgoing events. Based on events outside the upgoing muon peak, we estimate there are $9 \pm 5$ background events in the total data set. In addition to these events, we estimate that there are $8 \pm 3$ events which result from upgoing charged particles produced by downgoing muons in the rock near MACRO. Finally, it is estimated that $11 \pm 4$ events are the result of interactions of neutrinos in the very bottom layer of MACRO scintillator. A statistical subtraction from the data is made for these backgrounds prior to calculation of the flux. Hence, the observed number of upward, 
through-going muons integrated over all zenith angles is 451 .

A Monte Carlo has been used to estimate the expected number of upgoing muons. We use the Bartol neutrino flux [15], which has a systematic uncertainty of $\pm 14 \%$, taking into account the agreement with measurements of the flux of muons in the atmosphere. We use the Morfin and Tung parton set $\mathrm{S}_{1}$ [16] for calculation of the $\nu N$ cross section. These parton distributions were chosen based on good agreement of the resulting $\sigma_{T}$ compared to the world average at $E_{\nu}=100 \mathrm{GeV}$. We estimate a systematic error of $\pm 9 \%$ on the upgoing muon flux due to uncertainties in $\sigma(\nu N)$, including low-energy effects [17]. The energy loss for muons propagating through rock is taken from Lohmann et al. 18], adjusting the energy loss for the average composition of rock in the Gran Sasso. A $5 \%$ systematic uncertainty in the flux of upgoing muons results from this calculation due to uncertainties in the rock composition and uncertainties of muon energy loss. Adding in quadrature all the quoted errors results in a total systematic uncertainty of $17 \%$ on the expected flux which is almost uniform with zenith angle. The expected upgoing muon fluxes based on different neutrino fluxes 20 24 are within $10 \%$ of the value presented here. The detector has been simulated using GEANT [19], and simulated events are processed in the same analysis chains as the data. An efficiency factor of 0.97 is applied to the expected number of events based on various electronic efficiencies which have been explicitly measured using downgoing muons.

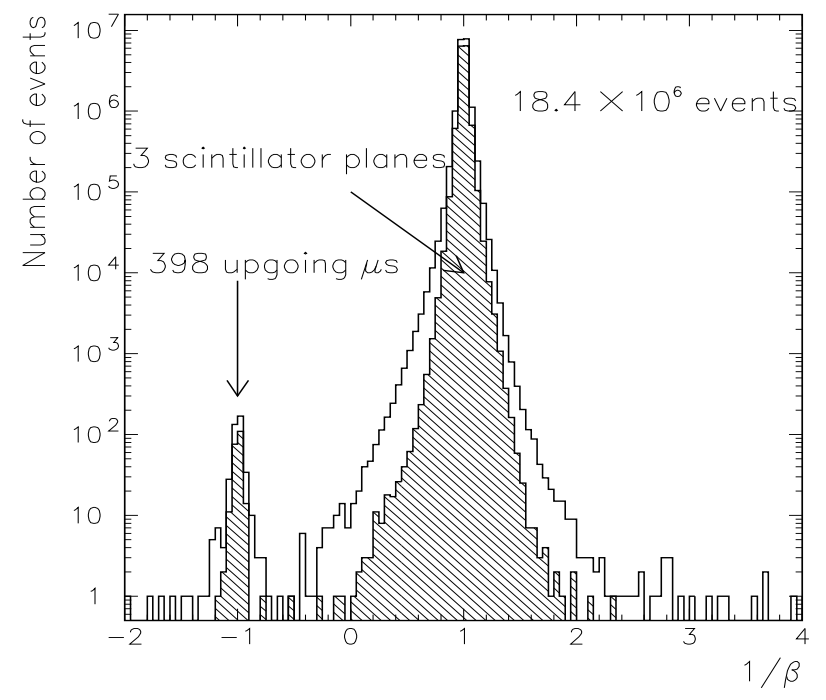

FIG. 1. Distribution of $1 / \beta$ for all muons in the data set taken with the full detector apparatus. A clear peak of upgoing muons is evident centered on $1 / \beta=-1$. The widths of the distributions for upgoing and downgoing muons are consistent. The shaded part of the distribution is for the subset of events where three scintillator layers were hit.

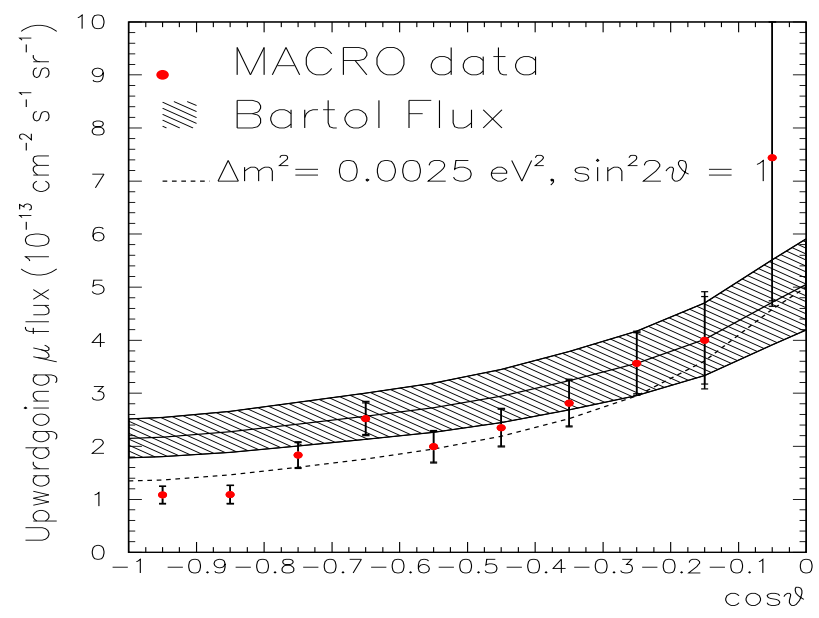

FIG. 2. Zenith distribution of flux of upgoing muons with energy greater than $1 \mathrm{GeV}$ for data and Monte Carlo for the combined MACRO data. The solid curve shows the expectation for no oscillations and the shaded region shows the uncertainty in the expectation. The dashed line shows the prediction for an oscillated flux with $\sin ^{2} 2 \theta=1$ and $\Delta m^{2}=0.0025$ $\mathrm{eV}^{2}$.

Care has been taken to ensure a complete simulation of the detector acceptance in the Monte Carlo and to minimize the systematic uncertainty in the acceptance. Comparisons have been made between several different analyses and acceptance calculations, including separate electronic and data acquisition systems. Studies have been made on trigger inefficiencies, background subtraction, streamer tube efficiencies, and efficiencies of all data quality cuts. Data distributions over many different variables (positions of events, azimuth angle, time distributions, etc.) have been studied and shown to be consistent with expectations. The sum (in quadrature) of all the systematic errors on the acceptance is $\pm 6 \%$ for the total number of events. The systematic uncertainty on the acceptance for zenith angle bins around the horizon is larger than near the vertical due to detector geometry effects and smaller statistics for downgoing muons.

The number of events expected integrated over all zenith angles is 612 , giving a ratio of the observed number of events to the expectation of $0.74 \pm 0.036$ (stat) \pm 0.046 (systematic) \pm 0.13 (theoretical). The probability to obtain a result at least as far from unity as this is 0.0003 if the Bartol Monte Carlo represents the true parent flux of neutrinos. However, taking into account the relatively large theoretical uncertainty on the flux (mostly on the normalization), the same probability is 0.14. Hence, there is a low probability that the Bartol neutrino flux represents the true flux of upgoing neutrinos at MACRO, but the uncertainty on the normalization of this flux makes it difficult to conclude (from this test alone) that new physics, such as neutrino oscillations, must be responsible for the discrepancy. 
Figure 2 shows the zenith angle distribution of the measured flux of upgoing muons with energy greater than $1 \mathrm{GeV}$ for all MACRO data compared to the Monte Carlo expectation for no oscillations (solid line) and with an oscillated flux with $\sin ^{2} 2 \theta=1$ and $\Delta m^{2}=0.0025 \mathrm{eV}^{2}$ (dashed line). The range for the Monte Carlo expectation for the unoscillated flux reflects the $\pm 17 \%$ systematic uncertainty in that prediction. The shape of the angular distribution is different than the expectation giving a $\chi^{2}=26.1$ for 8 degrees of freedom (probability of 0.001 for a shape at least this different from the expectation) for the case of no oscillations but with the number of events in the Monte Carlo normalized to the number in the data.

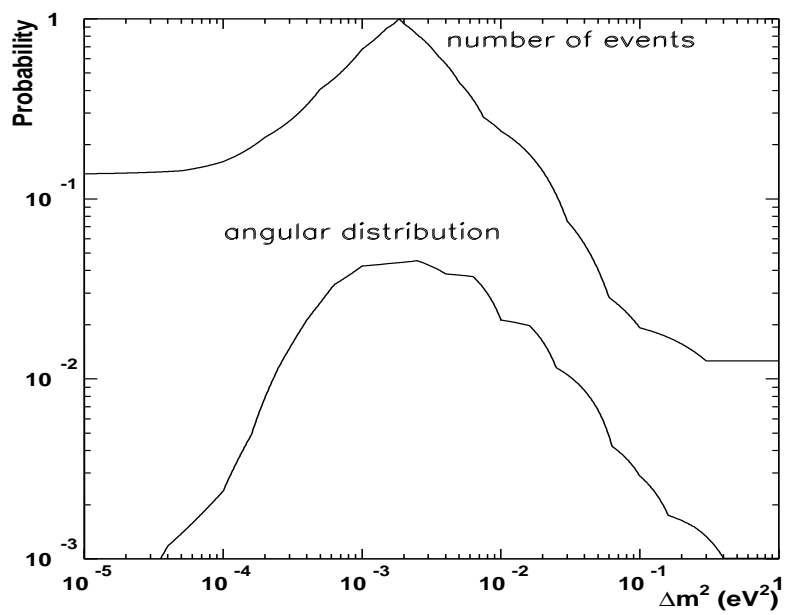

FIG. 3. Probabilities for obtaining the observed MACRO results on upgoing muons for $\nu_{\mu} \rightarrow \nu_{\tau}$ oscillations with $\sin ^{2} 2 \theta=1.0$ and for $\Delta m^{2}$ as shown. For the number of events, the curve shows the probability of observing a number of events which differs from the expectation by at least as much as the MACRO data for a given value of $\Delta m^{2}$. For the angular distribution, the curve shows the probability to observe a distribution which is at least as unlike the expectation based on a $\chi^{2}$ comparison of the shape of the data as a function of zenith angle.

To test oscillation hypotheses, we calculate the independent probability for obtaining the number of events observed and the angular distribution for various oscillation parameters. Figure 3 shows the probability of obtaining a number of events which differs from the expectation by at least as much as the MACRO observation for $\sin ^{2} 2 \theta=1.0$ and various $\Delta m^{2}$ for $\nu_{\mu} \rightarrow \nu_{\tau}$ oscillations. (This is a two-sided Gaussian probability.) The expectation for $\Delta m^{2}=0.002 \mathrm{eV}^{2}$ agrees with the observed number of events.

The probability of $\chi^{2}$ for obtaining the observed shape of the angular distribution has been computed as above for oscillation hypotheses and is also presented in Fig. 3 for $\nu_{\mu} \rightarrow \nu_{\tau}$ oscillations. The number of events under different flux hypotheses is always normalized to the observed number of events for this comparison. A maximum probability of $5 \%$ is obtained for a distribution at least this different from the expectation for $\nu_{\mu} \rightarrow \nu_{\tau}$ oscillations. This occurs for $\sin ^{2} 2 \theta=1.0$ and $\Delta m^{2}=0.0025$ $\mathrm{eV}^{2}$, but the probability is changed little within a decade of $\Delta m^{2}$ around this value. However, it is notable that the same best value for $\Delta m^{2}$ is obtained independently from both the angular distribution and the number of events. The somewhat low probability for any of these hypotheses is the result of the relatively low number of events in the region $-1.0<\cos \theta<-0.8$ compared to the number of events in the region $-0.8<\cos \theta<-0.6$.

Figure 14 shows probability contours for oscillation parameters using the combination of probability for the number of events and $\chi^{2}$ of the angular distribution. The best-fit point has a probability of $17 \%$. The solid lines show the probability contours for $10 \%$ and $1 \%$ of the bestfit value (i.e. $1.7 \%$ and $0.17 \%$ ). The dashed lines show the exclusion contours at the $90 \%$ and $99 \%$ confidence levels based on application of the Monte Carlo prescription of reference [25]. The "sensitivity" (not shown) is slightly larger than the curve for $10 \%$ of $P_{\max }$. The sensitivity is the $90 \%$ contour which would result from the preceding prescription if the data and Monte Carlo happened to be in perfect agreement at the best-fit point. It should be noted that this prescription for producing confidence-level intervals assumes that the hypothesis is correct.

Possible systematic effects have been studied and shown to be too small to explain the observed anomalous shape in the zenith distribution. The detector acceptance is best understood (from downgoing muons) near the vertical, where the biggest deviation compared to the Monte Carlo without oscillations is observed. The data from all running periods are consistent in the shape of the zenith distribution. We have compared the zenith distribution of down-going muons with a Monte Carlo expectation based on the known overburden; the two distributions agree well. We have compared the measured flux of downgoing muons using the same analysis as for the upgoing muons and find the result is consistent with expectations (see ref. 26]). The possibility of a waterfilled cavern below MACRO has been studied, although no such caverns are known to exist in the region of the Gran Sasso. If all of the region below MACRO were water, a maximum $15 \%$ depletion would be observed in the flux of upgoing muons. Any realistic water-filled cavern would result in a depletion of no more than about $5 \%$. For MACRO, we have shown that upgoing charged particles produced by downgoing muons contribute a background of $2 \%$ of the total number of upgoing muons [14]. This rate could be higher for experiments located in laboratories with less overburden than the Gran Sasso.

It has recently been suggested that oscillations between $\nu_{\mu}$ and a sterile $\nu$ could qualitatively produce a shape in the zenith distribution of upgoing muons similar to that observed by MACRO [27]. This would result from a matter effect in the center of the Earth. However, due to suppressed oscillation amplitude, the current model does 
not offer a better quantitative agreement with MACRO data than the $\nu_{\mu} \rightarrow \nu_{\tau}$ hypothesis, giving a maximum probability of $2 \%$.

In conclusion, we have reported on a measurement of the flux of upgoing muons, produced by neutrinos (with $<E_{\nu}>\sim 100 \mathrm{GeV}$ ) originating in atmospheric cosmicray showers. The ratio of the number of observed to expected events integrated over zenith angles from $-1.0 \leq$ $\cos \theta \leq-0.1$ is $0.74 \pm 0.036$ (stat) \pm 0.046 (systematic) \pm 0.13 (theoretical). The observed zenith distribution does not fit well with the expectation, giving a maximum probability for $\chi^{2}$ of only $0.1 \%$. The acceptance of the detector has been extensively studied using downgoing muons, independent analyses, and Monte-Carlo simulations. The remaining systematic uncertainties cannot be the source of the discrepancies between the data and expectations. We have investigated if the anomaly could be the result of neutrino oscillations. Both techniques independently yield mixing parameters of $\sin ^{2} 2 \theta=1.0$ and $\Delta m^{2}$ of a few times $10^{-3} \mathrm{eV}^{2}$. However, the observed zenith distribution does not fit well with any expectations, giving a maximum probability for $\chi^{2}$ of only $5 \%$ for the best oscillation hypothesis. We conclude that these data favor a neutrino oscillation hypothesis, but with unexplained structure in the zenith distribution not easily explained by either the statistics or systematics of the experiment.

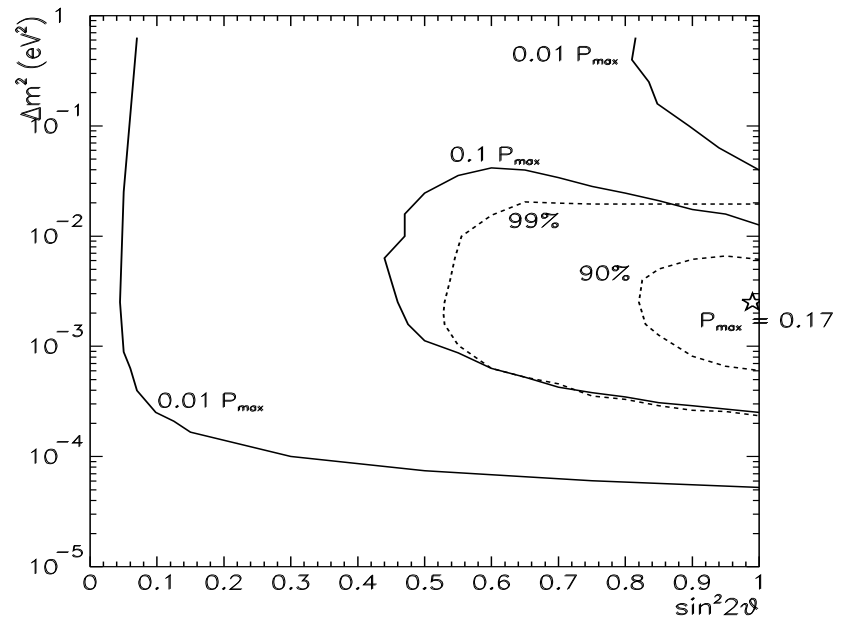

FIG. 4. Probability contours for oscillation parameters for $\nu_{\mu} \rightarrow \nu_{\tau}$ oscillations based on the combined probabilities of zenith shape and number of events tests. The best-fit point has a probability of $17 \%$ and iso-probability contours are shown for $10 \%$ and $1 \%$ of this value (i.e. $1.7 \%$ and $0.17 \%$ ). The dashed lines show exclusion confidence intervals at the $90 \%$ and $99 \%$ levels calculated according to reference 25. Since the best probability is outside the physical region the confidence intervals regions are smaller than the one expected from the sensitivity of the experiment. The "sensitivity" contour (not shown) is slightly larger than that for $10 \%$ of $P_{\max }$.

We are analyzing other topologies of neutrino events. We will publish shortly the complementary results from semi-contained events with the neutrino interaction within the detector $\left(<E_{\nu}>\sim\right.$ few $\left.\mathrm{GeV}\right)$ and upward going stopping muons [13].

We gratefully acknowledge the staff of the Laboratori Nazionali del Gran Sasso and the invaluable assistance of the technical staffs of all the participating Institutions. For generous financial contributions we thank the U.S. Department of Energy, the National Science Foundation, and the Italian Istituto Nazionale di Fisica Nucleare, both for direct support and for FAI grants awarded to non-Italian MACRO collaborators.

[1] IMB Collaboration, D. Casper et al., Phys. Rev. Lett. 66 (1991) 2561; R. Becker-Szendy et al., Phys. Rev. D46 (1992) 3720.

[2] Kamiokande Collaboration, K.S. Hirata et al., Phys. Lett. B205 (1988) 416; Phys. Lett. B280 (1992) 2.

[3] Kamiokande Collaboration, Y. Fukuda et al., Phys. Lett. B335 (1994) 237.

[4] Super-Kamiokande Collaboration, preprint hep-ex/9805006 "Study of the atmospheric neutrino flux in the multi-GeV energy range", preprint hep-ex/9803006 "Measurement of a small atmospheric muonneutrino/electron-neutrino ratio" submitted to Phys. Lett. B.

[5] Soudan 2 Collaboration, W.W. M. Allison et al., Phys. Lett. B391 (1997) 491; T. Kafka, 5th TAUP Workshop proceedings, Gran Sasso, Italy, 1997.

[6] Frejus Collaboration, Ch. Berger et al., Phys. Lett. B227 (1989) 489; K. Daum et al., Z. Phys. C66 (1995) 417.

[7] NUSEX Collaboration, M. Aglietta et al., Europhys. Lett. 8 (1989) 611; 23rd ICRC proceedings, Calgary, Vol. 4 (1993) 446.

[8] Baksan Collaboration, S. Mikheyev, 5th TAUP Workshop proceedings, Gran Sasso, Italy, 1997.

[9] Kamiokande Collaboration, Y. Totsuka et al., Nucl. Phys. B31 (Proc. Suppl.) (1993) 428; M. Mori et al., Phys. Lett. B270 (1991) 89.

[10] IMB Collaboration, R. Becker-Szendy et al., Phys. Rev. Lett. 69 (1992) 1010; Nucl. Phys. B38 (Proc. Suppl.) (1995) 331.

[11] MACRO Collaboration, S. Ahlen et al., Nucl. Instr. and Meth. A324 (1993) 337.

[12] MACRO Collaboration, S. Ahlen et al., Phys. Lett. B357 (1995) 481; "Neutrino Induced Upward-going muons with the MACRO Detector", Neutrino 96 conference proceedings, Helsinki, 1996; "The measurement of the atmospheric muon neutrino flux using MACRO", INFN/AE97/21 and Proc. of the 25th ICRC, Durban, Vol. 7 (1997) 41.

[13] MACRO Collaboration, "Upward-going muons and MACRO", presented by F. Ronga at Neutrino 98, Takayama, Japan, 1998 (to be published in conference proceedings).

[14] MACRO Collaboration, M. Ambrosio et al., "The ob- 
servation of upgoing charged particles produced by high energy muons in underground detectors", accepted for publication in Astroparticle Physics.

[15] V. Agrawal, T.K. Gaisser, P. Lipari and T. Stanev, Phys. Rev. D53 (1996) 1314.

[16] J. G. Morfin and W. K. Tung, Z. Phys. C52 (1991) 13.

[17] P. Lipari, M. Lusignoli and F. Sartogo, Phys. Rev. Lett. 74 (1995) 4384.

[18] W. Lohmann et al., CERN-EP/85-03 (1985).

[19] R. Brun et al., "GEANT", CERN report DD/EE84-1 (1987).

[20] A.V. Butkevich et al., Sov. J. Nucl. Phys. 50 (1989) 90.

[21] K. Mitsui et al., Nuovo Cimento 9C (1986) 995.

[22] L. V. Volkova, Sov. J. Nucl. Phys. 31 (1980) 1510.

[23] M. Honda et al., Phys. Rev. D52 (1995) 4985.

[24] G. Battistoni et al., "A new calculation of the atmospheric neutrino flux: The FLUKA approach", Submitted to Nucl. Phys. B (1998).

[25] G. Feldman and R. Cousins, Phys. Rev. D57 (1998) 3873.

[26] MACRO Collaboration, M. Ambrosio et al., Phys. Rev. D52 (1995) 3793.

[27] Q.Y. Liu and A.Y. Smirnov, preprint IC-97-211, hep$\mathrm{ph} / 9712493$. 